\title{
N $92-11062$
}

\section{A Method for Interference Mitigation in Space Communications Scheduling}

\author{
Yen $\mathrm{F}$. Wong \\ James L. Rash \\ NASA Goddard Space Flight Center \\ Greenbelt, Maryland
}

\begin{abstract}
Increases in the number of user spacecraft and data rates supported by NASA's Tracking and Data Relay Satellite System (TDRSS) in the S and Ku bands could result in communications conflicts due to mutual interference. More attention must be paid to this problem in terms of communications scheduling. A method to mitigate interference while minimizing unnecessary scheduling restrictions on both TDRSS network and user resources, based on consideration of all relevant communications parameters, has been developed. The steps of this method calculate required separation angles at TDRS and produce potential interference intervals. which can be used in the production of schedules free of unacceptable interference. The method also can be used as the basis for analysis, evaluation. and optimization of user schedules with respect to communications performance. This paper describes the proposed method and its potential application to scheduling in space communications. Test cases relative to planned missions, including Earth Observing System, Space Station Manned Base, and Space Shuttle, are discussed.
\end{abstract}

\section{Introduction}

Scheduling of user spacecraft communications utilizing the geosynchronous data relay satellites of NASA's Tracking and Data Relay Satellite System (TDRSS) (Figure 1) must increasingly be concerned with the effects of mutual interference between users. While current techniques for interference mitigation are adequate for scheduling under light system loading, the concerns regarding mutual interference will become more serious with projected increases in loading, especially in the late 1990s and beyond (NASA Goddard Space Flight Center, forthcoming).

Consideration of the effect of communications factors such as signal to interference Consideration of the effect of communications factors is beyond the scope of current
ratio (S/I), BER margin degradation, and power received ing
TDRSS network scheduling systems.

Furthermore, link margins are always constrained to the minimum acceptable value by the high costs assoclated with designing, building, and orbiting spacecraft with higher margins. Consequently, mutual interference would become more likely, and would tend to be more serious whenever it should occur.

The ultimate objective is to schedule interference-free communications while minimizing constraints imposed both on user spacecraft missions and on the use of TDRSS resources. This objective cannot be accomplished absent the capability to analyze, evaluate, and optimize user schedules with respect to communications performance.

The Communications Link Analysis and Simulation System (CLASS) developed by NASA Goddard Space Flight Center (GSFC) is a software tool for the prediction and evaluation of TDRSS/user spacecraft communications link performance. CLASS is a unique system designed to consider all communications channel parameters that affect link performance, including interference (NASA Coddard Space Flight Center, 1989, September).

The need for a capability that considers all relevant communications parameters in the analysis, evaluation, and optimization of user schedules relative to mutual interference has led to the development of such a capability within CLASS. 


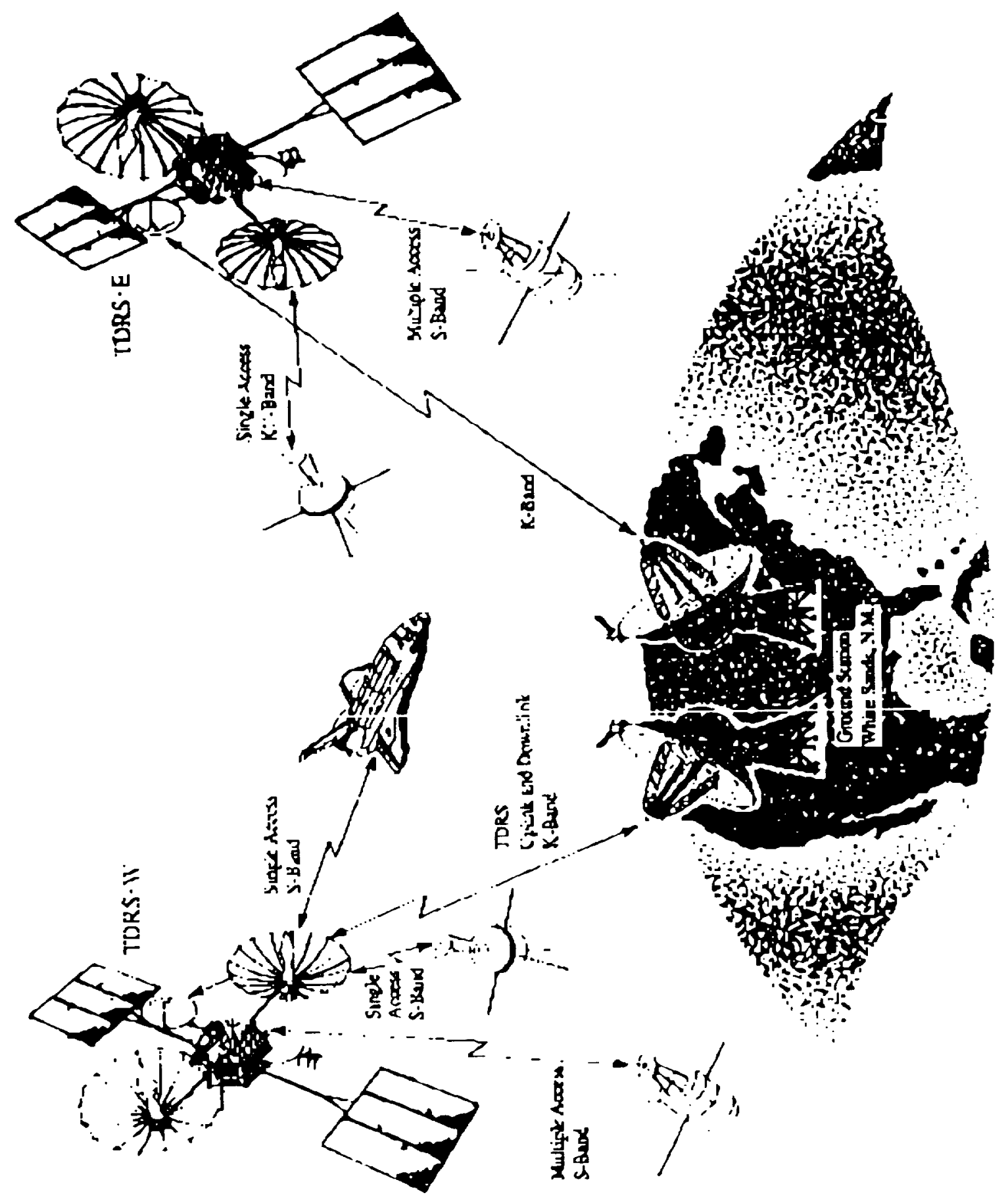

Figure 1. TDRSS Configuration. 
An overview of TDRSS telecommunications is presented in the next section. The subsequent sections describe the proposed approach and present lllustrative test cases. A discussion of results is then offered, along with a summary and an indication of directions for future work.

\section{TDRSS Telecommunications Overview}

NASA's Tracking and Data Relay Satellite System consists of a space segment and a ground segment as shown in Figure 1. The ground segment of TDRSS consists of a ground terminal at White Sands. New Mexico. The operational space segment consists of a user Iransponder on each user spacecraft, and three in-service satellites in geostationary orbit at 41,171 , and 174 degrees west longitude. In the future, a cluster of two TDRS's 3 degrees apart may be placed in operation at each of the approximate positions of 41 and 171 degrees west longit ude.

TDRSS provides telecommunications in $S$ band via single access (SA) and multiple access (MA) service, and in Ku band via the SA service. Forward links (signals from ground station via TDRS to user) operate at data rates from $0.1 \mathrm{Kbps}$ to $25 \mathrm{Mbps}$, and return links (signals from user to ground station via TDRS) operate at data rates from $0.1 \mathrm{Kbps}$ to 300 Mbps (NASA Goddard Space Flight Center, 1988, September).

Each TDRS may support a maximum of five forward links: two S-band and two K-band on each of the two SA antennas and one S-band on the MA system. By design, each TDRS may support a maximum of 24 return links: 20 on the MA antenna, two at S-band on SA (SSA), and two at K-band on SA (KSA). Future TDRS cluster operations will approximately double the resources available at each of the two geostationary positions at 41 and 171 degrees west longitude.

The information necessary to characterize the communication systems of TDRSS and user spacecraft, such as antenna type, coding scheme, data rate, signal level, or polarization, as well as the channel environments, is maintained in CLASS data bases. All possible sources of effects on the RF signal are taken into account. including vehicle and earth multipath, vehicle blockage, atmospherics, and signal reflections from terrestrial surfaces.

\section{Interference Analysis Considerations}

Since all TDRS forward links are PN spread, and since the data rates are less than or equal to $300 \mathrm{Kbps}$, the PN processing gain over interference will be at least $10 \mathrm{~dB}$. Therefore, interference on destred user forward channels can be neglected.

The interference problem between two return links is more complicated because data rates on return links in general are much higher than on forward links, and because the links may or may not be PN spread and may or may not be cross polarized. Hence, in this paper, interference mitigation is concerned only with the user return channel.

The problem of multiple simultaneous interferers is not considered in this paper.

\section{A Model for Communications Performance in the Presence of Mutual Interference}

The proposed approach to interference miligation uses BER margin degradation, formulated as a function of signal to interference level ratio (S/I), as the basic parameter for determination of channel communications performance for a link in the presence of interference (Bhargava, 1981). BER margin degradation includes all the factors in the ground receiver (data rate (bandwidth) difference between the desired user and interferer, and implementation loss), and fully reflects channel performance when interference exdsts.

Figure 2 shows the relationship between BER degradation and $S / I$ in a representative case.

Nonnegative BER margin is considered to correspond to acceptable communications performance when a link is degraded by interference. In general, degradation is computed by simulation, with S/I as an input parameter. 


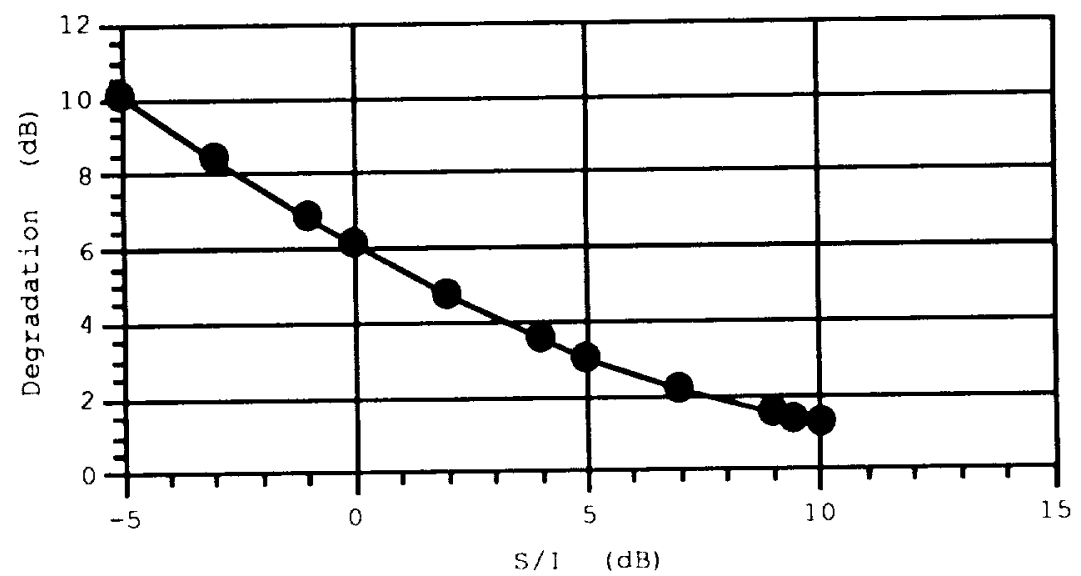

Figure 2. Computed relationship between degradation and $S / l$ for the case where the desired user is the Space Shuttle Orbiter using channel 3,50 Mbps coded, and the interferer (assumed to be on the TDRS SA antenna boresight) is Space Station Freedom using the 50 Mbps (1+Q) link. The desired user and interferer links are cross polarized with an assumed polarization rejection of the interfering signal of $15 \mathrm{~dB}$ on the TDRS SA antenna boresight.

The signal to interference level ratio $S / I$ in $\mathrm{AB}$ at TDRS is defined as a function of the separation angle $\alpha$ between the desired user and the interferer as seen from TDRS:

$$
\underline{S_{I}}(\alpha)=\left(P_{d}+G(0)\right)-\left(P_{t}+G(\alpha)+R(\alpha)\right)+G_{p}+A_{p}+L_{j s}
$$

where

$P_{d}=$ the worst case (maximum range) TDRS received power at unlty antenna gain for the desired user (in $\mathrm{dB}$ ) including the loss due to the nonperfect polarization match between the TDRS and desired user antennas. It is assumed that the desired user is on the TDRS antenna boresight and that the desired user's antenna is pointing toward TDRS. $P_{d}$ includes contributions from stochastic sources such as multipath (vehicle, earth. and atmospheric) and RFI.

$P_{t}=$ the best case (minimum range) TDRS received power at unity antenna gain for the interferer (in $\mathrm{dB}$ ).

$\mathrm{G}=$ the TDRS antenna gain (in $\mathrm{dB}$ ) as a function of the angle $\alpha$.

$\mathrm{R}=$ the polarization rejection of the interferer's signal at the TDRS antenna (in $\mathrm{dB}$ ) as a function of angle $\alpha$. R always has a negative value when rejection is present (interferer oppositely polarized), and is zero otherwise.

$G_{p}=10^{*}$ ALOG 10 (Desired user PN chip rate/Desired channel symbol rate) is the processing gain (in $\mathrm{dB}$ ) of the PN spread signal

$A_{p}=10^{*}$ ALOG10 (Interferer channel PN chip rate/Desired channel symbol rate) is the reduction factor (in $\mathrm{dB}$ ) if the interferer is PN spread when the desired channel is not PN spread.

$L_{\text {s }}=$ interferer power reduction $(\mathrm{in} \mathrm{dB})$ due to frequency separation.

$L_{f s}$ applies to cases (for example, non-TDRS user spacecraft from European or other space agencies) where the frequency separation between the desired user and the interferer is small (less than $10 \mathrm{MHz}$ ) but nonzero. Under the current TDRSS design, any two different TDRS-user transmitting frequencles are separated by at least $10 \mathrm{MHz}$ : in such cases, degradation of a desired signal due to a single interfering signal may be neglected.

In case neither desired user nor interferer is PN coded. the adjustment after the match filter at the ground terminal due to a large bandwidth (data rate) difference is included in the degradation calculation. 
For GPSK modulation, S/I is calculated for all channels, and since the channel having the smallest S/I will suffer most from interference, the minimum S/I value is used.

Since among the above terms only $G$ and $R$ are functions of $\alpha$. Equation (1) leads immediately to the following for any given $\alpha_{1}$ and $\alpha_{2}$, each an allowed value of $\alpha$ :

$$
\frac{S}{I}\left(\alpha_{2}\right)-\frac{S}{I}\left(\alpha_{1}\right)=-[G(\alpha)+R(\alpha)]_{\alpha=\alpha_{1}}^{\alpha=\alpha_{2}}
$$

which expresses the fact that for a given change in $\alpha$. the change in $S / I$ equals the change in the negated adjusted antenna gain: $\Delta(S / I)=-\Delta(G+R)$.

The negated TDRS antenna gain pattern envelope, without polarization adjustment, is shown in Figure 3(a), modeled to represent the main beam, the first null, the peak of the first sidelobe, and a logarithmic relation for the remainder of the pattern.

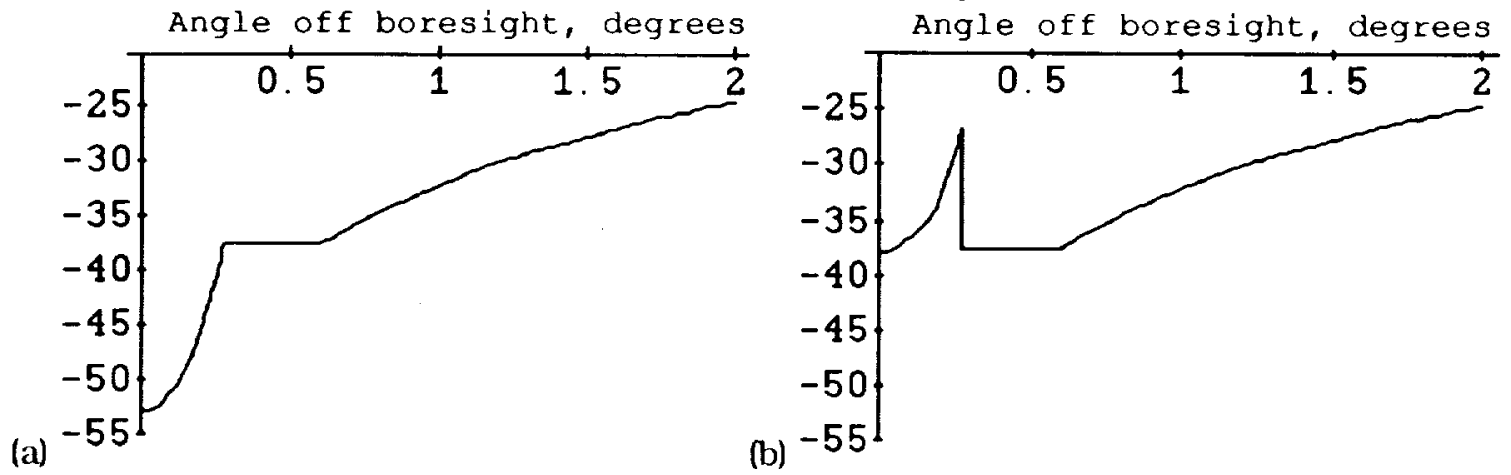

Figure 3. Negated TDRS SA antenna gain pattern envelope: (a) without polarization adjustment, and (b) adjusted by the polarization rejection of a cross polarized signal from an antenna having an axial ratio of $2.1 \mathrm{~dB}$. Note that in both cases, the global minimum of the curve occurs at boresight $(\alpha=0)$.

Figure 3(b) shows an example of the negated adjusted antenna gain, $-[G+R]$, in which the gain of the TDRS SA antenna is adjusted by the polarization rejection of an oppositely polartzed user antenna. (A formulation of polarization rejection at, and a model of the axtal ratlo of, the TDRS SA antenna are presented in the Appendix.) The transmitting antenna on the Interferer (Space Station Manned Base (SSMB)) has a boresight axial ratio of $2.1 \mathrm{~dB}$ (a calculated value based on the assumption that the recetving TDRS antenna has a boresight axial ratio of $1 \mathrm{~dB}$ and polarization rejection of $15 \mathrm{~dB}$ ).

In general, the negated adjusted antenna gain curve, $-|G+R|$, will have multiple relative minima. The global minimum value of the curve may correspond to more than one value for the separation angle $\alpha$ (interferer's angle off boresight). We let $\dot{\alpha}^{*}$ denote the least such value of $\alpha$.

Of course, it is possible for $\alpha^{*}$ to be zero. Indeed, if the interferer has the same polarization as the desired user, the polarization rejection at the TDRS SA antenna is zero for all $\alpha$, so that the negated antenna gain (Figure 3(a)) has its global minimum at boresight (under the normal assumption that the antenna gain envelope has its global maximum at boresight). Hence, in this case, $\alpha^{*}=0$.

When the interferer and desired user are cross polarized, it is still possible for $\alpha^{\circ}$ to be zero, depending on the exact nature of the model used to represent the polarization rejection R. Figure 3(b) illustrates such a possibility, for the case where the interferer is SSMB. As shown in the figure. the global minimum of the adjusted antenna gain $-[G+R]$ occurs at zero degrees off boresight, so that $\alpha^{\circ}=0$ in this example.

From Equation (2), S/I can be expressed as follows:

$$
\frac{S}{I}(\alpha)=-[G(\alpha)+R(\alpha)]+\frac{S}{I}(0)+[G(0)+R(0)]
$$


that is, the form of $S / 1$, as a function of angle off boresight, is merely that of the negated adjusted antenna gain, shifted vertically by a constant (the boresight value of $S / I$ plus the boresight value of $[G+R]$ ). The graph of $S / I$ is shown in Figure 4 for representative cases (to be described later, in Table 4).

From the S/I graph, it is possible to find a separation angle between the user spacecraft such that no unacceptable interference can occur. This is the basis of the method proposed in this paper for mutual interference mitigation.
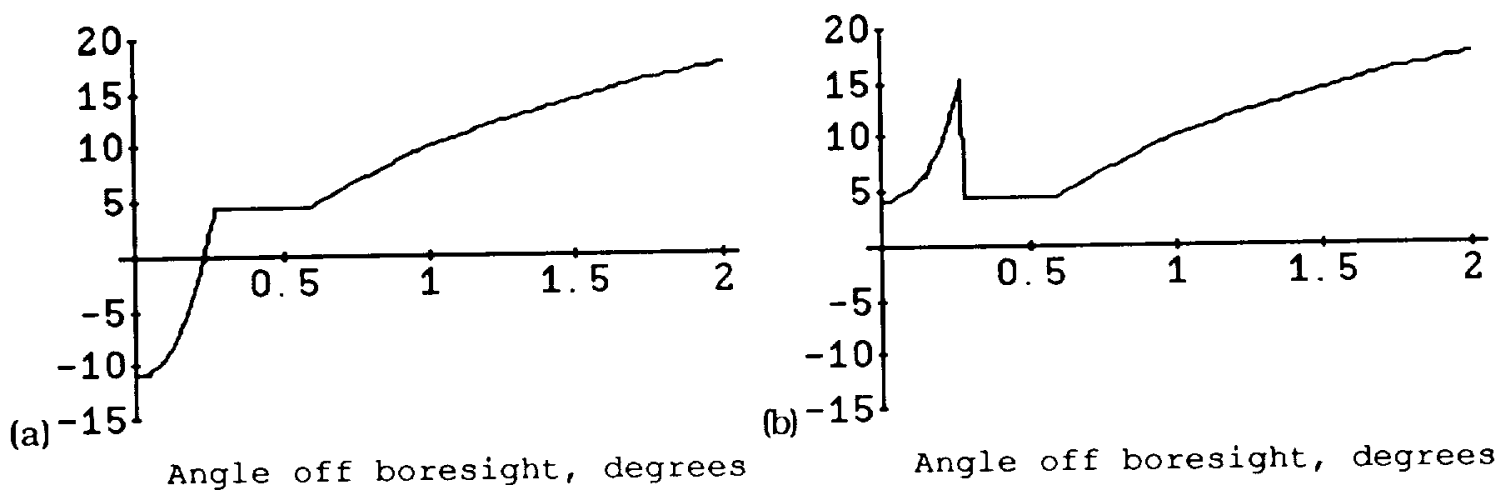

Figure 4. S/l as a function of interferer's angle off boresight: (a) for the case where desired user and interferer have the same polarization, and (b) for a representative case where desired user and interferer are oppositely polarized, showing the effect, on the interfering signal, of TDRS antenna gain and polarization rejection.

\section{Worst S/I}

As the interferer moves of boresight, the interferer's power $P_{1}$ changes in a manner dictated by the negated adjusted antenna gain curve. When $-[G+R]$ reaches a local minimum. the interferer's power reaches a local maximum. Since the desired user remains on boresight. $P_{d}$ remains constant. Therefore, when $-[G+R]$ reaches its global minimum (e.g., at $\alpha=\alpha^{*}$ ). the value of $S / I$ will also reach its global minimum. This minimum S/I is the "worst $S / I^{\prime \prime}$, and so we have, by Equation (3):

$$
\left(\frac{S}{I}\right)_{\text {worst }}=\frac{S}{I}(\alpha)=-\left[G\left(\alpha^{*}\right)+R\left(\alpha^{*}\right)\right]+\frac{S}{I}(0)+[G(0)+R(0)]
$$

Note that if $\alpha^{\circ}=0$,

$$
\left(\frac{S}{I}\right)_{\text {worst }}=\frac{S(0)}{I}
$$

\section{Required S/I}

The required $S / I$ is defined as the value of S/I such that the degradation of the desired user signal equals the worst case channel margin. Computer simulation is used to obtain the required S/I for any given combination of desired user and Interferer links. The worst S/I may or may not be less than the required S/I. If the worst $S / I$ is less than the required $S / I$. then unacceptable mutual interference is possible for some possible separation angles. 


\section{Interference Mitigation via Separation Angle and Potential Interference Intervals}

\section{Required separation angle}

Since the desired user is assumed to be on the TDRS antenna boresight, and since antenna gain decreases off boresight, a sufficient variation of the interferer's separation angle provides discrimination between the signals, reduces the interference level, and increases the $S / I$ level ratlo. In the case where the required $S / I$ is greater than the worst $S / I$ (1.e., where interference is possible) the required $S / I$ corresponds to certain separation angles, which can be read directly from the graph of S/I (see Figure 4). Note that due to the possibility of multiple lobes in the adjusted antenna gain graph (and therefore multiple lobes in the graph of S/I) there may be multiple disjoint ranges of separation angle providing at least the necessary value of $S / I$. The largest of all the angles where $S / I$ is equal to the required $S / I$ is defined as the required separation angle. Any separation angle not less than this angle assures an acceptable level of interference.

\section{Potential interference intervals}

A potential interference interval is defined as any time interval during which the separation angle between the two user spacecraft is less than the required separation angle as described above. During such intervals, unacceptable interference could occur if the given pair of links of the two spacecraft are active. The potential interference intervals, therefore, would constrain any interference mitigation scheduling process by specifying when the two links should not be used simultaneously for communications. How to decide which of the two links should not be scheduled during any potential interference interval is part of the algorithm used by the scheduler and is beyond the scope of this paper.

Potential interference intervals are calculated in a straightforward manner, based on given user orbital parameters and the required separation angle.

\section{A Procedure for Interference Mitigation in Scheduling}

A procedure is suggested for producing schedules free of unacceptable interference while minimizing restrictions on use of network and user resources. This procedure is based on a model for communications performance in the presence of interference, on required separation angle, and on potential interference intervals. It is summarized by the following
steps:

(1) For every pair of desired and interfering signals, determine $-[G+R]$ as a function of $\alpha$ (the separation angle at a given TDRS between the desired user and the interferer) where $G$ is the TDRS antenna gain envelope and $R$ is the polarization rejection of the interfering signal at the TDRS antenna. $\mathrm{R}$ is assumed to be zero if the desired user and interferer have the same polarkation.

(2) For every pair of desired and interfering signals, determine the least separation angle at which the function $-[G+R]$ has its global minimum value. Denote this angle $\alpha^{*}$.

(3) For every pair of desired and interfering signals, determine S/I, the signal to interference level ratio, as a function of $\alpha$ given by Equation (1) above. Calculate $(S / I)\left(\alpha^{\circ}\right)=(S / I)(0)-[G+R]\left(\alpha^{\circ}\right)+[G+R](0)$. This is the worst S/I. 
(4) For every pair of desired and interfering stgnals, determine by computer simulation the degradation of the desired signal that corresponds to $\mathrm{S} / \mathrm{I}\left(\alpha^{\circ}\right)$. This is the desired signal's worst degradation. Identlly all stgnal palrs where the desired signal's worst degradation exceeds the desired user's worst link margin. Mutual interference will be unacceplable for these signal pairs.

(5) For every pair of desired and interfering signals where interference is unacceptable as determined in step (4), determine by computer simulation the required $S / I$, i.e., the $S / I$ for which the degradation is equal to the desired signal's worst link margin.

(6) For every pair of desired and interfering signals where interference is unacceptable as determined in step (4), calculate the required separation angle (the largest separation angle between the desired user and interferer that provides the required $S / I$ as determined in step (5)).

(7) For every pair of desired and interfering signals where interference is unacceptable as detemined in step (4), and on the basis of the separation angles obtained in step (6). find all potential interference intervals, that is, intervals during which unacceptable interference is possible.

(8) Use the potential interference Intervals from step (7) as a constraint to a scheduler for generating schedules free of unacceptable interference. The effect of this constraint is to preclude the scheduling of any combination of desired/interferer links during any potentlal interference interval assoclated with that comblnation of links.

The first four steps can be used as a screening process to isolate the cases where unacceptable interference could occur. Steps (5) and (6) would be applied in such cases, as an intermediate process prior to execution of a scheduling system. Step (7) would be performed prior to every run of an interference mitigation scheduling system (step (8)).

\section{Implementation}

Software to produce potent lal interference intervals has been implemented within the CLASS environment as an intial step toward development of a scheduling system (illustrated in Figure 5) that incorporates the interference mitigation methodology described in this paper.

The principal components of the software are the analysis system. the required separation angle calculator, and the potential interference interval calculator. Each of these elements accesses the "interference analysis table".

The analysis system accesses CLASS data bases contalning link parameters (e.g., data rate, coding scheme, polarization, power), orbital elements, et cetera, in order to calculate required $S / I$, worst $S / I$, and worst degradation. These calculated values are stored into the interference analysis table for use by the required separation angle calculator. The required separation angle calculator also takes input from a file containing orbit and view period data.

Output from the required separation angle calculator is written into the interference analysis table.

The potential interference interval calculator reads the required separation angles and calculates all intervals during which every pair of potentially-interfering spacecraft have a separation less than the required separation angle. The potential interference intervals are written into a file, which can then be used as input to a scheduler.

Each line (record) in the interference analysis table consists of the following flems:

(1) Desired User ID 
(2) Desired User Link ID

(3) Desired User Channel

(4) Desired User Polarization

(5) Interferer ID

(6) Interferer Link ID

(7) Interferer Polarization

(8) Interferer Antenna Boresight Axial Ratio

(9) TDRS SA Antenna ID

(10) Desired User Worst Case Link Margin

(11) Required S/I

(12) Worst S/I

(13) Worst Degradation

(14) Required Separation Angle

IMSS Block Diagram

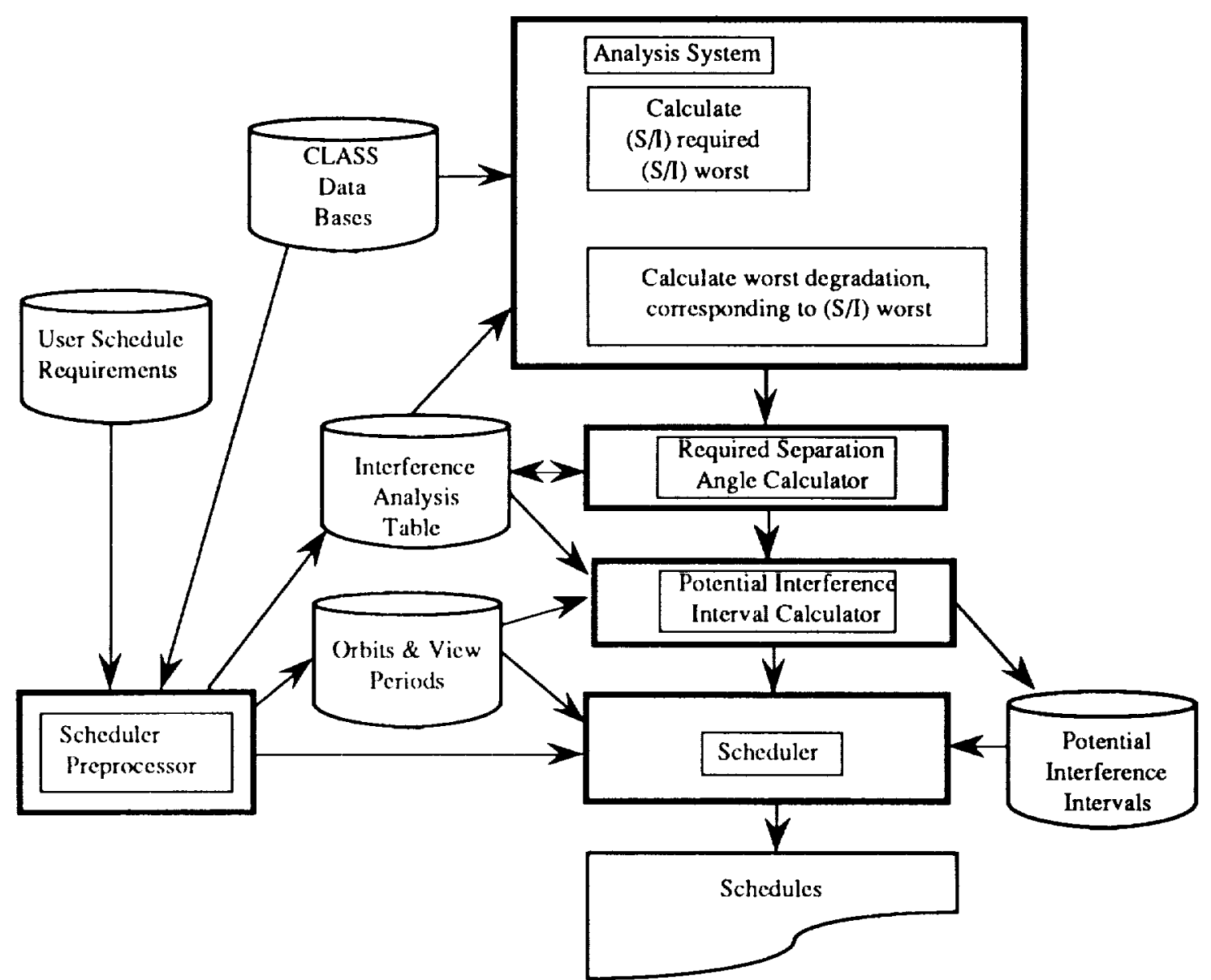

Figure 5. Block diagram of the proposed interference mitigation scheduling system (IMSS). The modules represented by the shaded blocks produce the potential interference intervals, and have been implemented in Goddard Space Flight Center's Communications Link Analysis and Simulation System (CLASS). 


\section{Application of the Approach: A Numerical Example}

The proposed interference mitigation approach has been applied to planned missions Including Space Shuttle Orblter (SSO). Space Station Manned Base (SSMB), and Earth Observing System (EOS).

The relevant communications parameters for these three missions, as obtained from an internal GSFC memorandum (NASA/GSFC, 1989, January 30) concerning Shuttle links. and from RF Interface Control Documents (NASA/GSFC, 1989, October 27: NASA/GSFC, 1990, February), are presented below.

All the missions in this example operate at Ku band with carrier frequency equal to $15.0034 \mathrm{GHZ}$, unspread.

SSO operates with Right Circular Polarization (RCP). Table 1 presents the link characteristics.

Table 1. Space Shuttle Orbiter Link Characteristics

\begin{tabular}{|l|c|c|c|}
\hline CHANNEL & $\begin{array}{c}\text { DATA RATE } \\
(\mathrm{kbps})\end{array}$ & $\begin{array}{c}\text { EIRP } \\
(\mathrm{dBW})\end{array}$ & $\begin{array}{c}\text { LINK MARGIN } \\
(\mathrm{dB})\end{array}$ \\
\hline Channel 1: Subcarrier Q & 192 & 39.4 & 19.0 \\
\hline Channel 2: Subcarrier I & 2,000 & 43.6 & 13.5 \\
\hline Channel 3: Baseband & 50,000 & 51.0 & 1.5 \\
\hline
\end{tabular}

Channels 1 and 2 are rate $1 / 2$ convolutional coded and channel 3 is uncoded.

SSMB operates with Left Circular Polarization (LCP) at data rates of $300 \mathrm{Mbps}$ and 50 Mbps. Table 2 presents the link characteristics.

Table 2. Space Station Manned Base Link Characteristics

\begin{tabular}{|c|c|c|c|}
\hline CHANNEL & $\begin{array}{c}\text { DATA RATE } \\
\text { (MbDS) }\end{array}$ & $\begin{array}{c}\text { EIRP } \\
\text { (dBW) }\end{array}$ & $\begin{array}{c}\text { LINK MARG IN } \\
\text { (dB) }\end{array}$ \\
\hline I & 150 & 57.1 & 3.0 \\
$Q$ & 150 & 57.1 & 3.0 \\
\hline$I$ & 25 & 57.1 & 10.8 \\
$Q$ & 25 & 57.1 & 10.8 \\
\hline
\end{tabular}

The parameters given above for SSMB are preliminary and subject to change.

EOS operates with RCP at data rates of $300 \mathrm{Mbps}$. Table 3 presents the link characteristics.

Table 3. Earth Observing System Link Characteristics

\begin{tabular}{|c|c|c|c|}
\hline CHANNEL & $\begin{array}{l}\text { DATA RATE } \\
\text { (MbPS) }\end{array}$ & $\begin{array}{l}\text { EIRP } \\
(\mathrm{dBW})\end{array}$ & $\begin{array}{l}\text { LINK MARGIN } \\
\text { (dB) }\end{array}$ \\
\hline $\mathrm{I}$ & 150 & 57.6 & 3.6 \\
$\mathrm{Q}$ & 150 & 57.6 & 3.6 \\
\hline
\end{tabular}

Interference analysis results

Table 4 presents the results of interference analysis. In Case 1, where the SSO (COLUMBIA) channel 3 (50 Mbps) experiences interference from the EOS $300 \mathrm{Mbps}$ link $(I+\mathrm{g})$, the required $S / I$ exceeds the worst $S / I$ by $17.8 \mathrm{~dB}$. The required separation angle 10 mitigate interference, which can be obtained directly from the appropriate S/I graph (Figure 4(a), with a required $S / I$ of $6.2 \mathrm{~dB}$ ), is 0.74 degrees. There is no unacceptable interference for SSO channels 1 and 2 . 
Table 4. Interference analysis table.

\begin{tabular}{|c|c|c|c|}
\hline \multirow{2}{*}{\multicolumn{2}{|c|}{ (2) }} & & \\
\hline & & Case 1 & Case 2 \\
\hline \multirow[t]{4}{*}{ Desired User } & User ID & COLUMBIA & COLUMBIA \\
\hline & Channel & $\mathrm{z}$ & 2 \\
\hline & Polarization & RHC & RHC \\
\hline & Worst Case Margin & 1.5 & 1.5 \\
\hline \multirow[t]{3}{*}{ Interferer } & User ID & EOS & SSMB \\
\hline & Polarization & RHC & LHC \\
\hline & Axial Ratio (dB) & 1.5 & $2.1 \star$ \\
\hline TDRS & SA Antenna ID & DEF LT & DEFLT \\
\hline \multirow[t]{2}{*}{$S / I$} & Required (dB) & $6.2 \star \star$ & $9.0 \star \star$ \\
\hline & Worst (dB) & -11.6 & 4.0 \\
\hline \multicolumn{2}{|c|}{ Worst Degradatation (dB) } & $\star \star$ & $\star \star$ \\
\hline \multicolumn{2}{|c|}{ Reguired Separation Angle (deg) } & 0.74 & 0.92 \\
\hline
\end{tabular}

*NOTE: In this case, the axial ratio for the interferer's antenna is a calculated value based on an assumed value of 15 dB for the polarization rejection of the interferer's link on the boresight of the TDRS SA antenna.

* NOTE: Obtained by computer simulation.

In Case 2, in which the interferer is the SSMB 50 Mbps link $(I+Q)$, the required S/I, 9.0 $\mathrm{dB}$, is greater than the worst $\mathrm{S} / \mathrm{I}$ by $5.5 \mathrm{~dB}$, and from Figure $4(\mathrm{~b})$ the required separation angle is 0.96 degrees. There is no unacceptable interference for SSO channels 1 and 2.

There is no unacceptable interference between the SSMB $300 \mathrm{Mbps}$ link $(I+Q)$ and the SSO channels 1,2 , and 3 .

\section{Potential Interference Intervals}

Potential interference intervals depend closely on the choice of orbits for user spacecraft. Figure 6 illustrates this dependency by showing the intervals for two choices for the user orbital elements. The only difference between these cholces is the value for the mean anomaly. For the choice tllustrated in Figure 6 (a), the difference in the mean anomaly is 0 degrees, and for the choice illustrated in Figure 6 (b). It is 20 degrees. The total of the potential interference intervals goes from $100 \%$ of the in-view time (Figure 6 (a))--approximately 813 minutes during the 24 hour scheduling period--to approximately 61 minutes (Figure 6 (b)). Thus, the potential interference intervals become shorter and less numerous as the orbital spacing of the users increases. Indeed, whenever the mean anomalies differ by more than approximately __ degrees, with all other factors remaining the same, unacceptable interference becomes impossible and potentlal interference intervals no longer exist.

\section{Application of Potential Interference Intervals in Existing Scheduling Systems}

Potential interference intervals also can be used in analyzing, evaluating and optimizing user schedules generated by current scheduling systems with respect to communications performance. The simultaneous communnications contacts specifled in any given schedule, produced by any scheduling system, can be compared with the potential interference intervals produced by the above procedure, in order to discover interference problems. Each such problem could then be evaluated relative to actual mission needs, priorities, or other aspects of the scheduling process. If a schedule revision is decided upon, by either a manual or an automated procedure, it could also be similarly checked and evaluated. Further, the degree to which schedules are free of mutual interference based on the potential interference intervals discussed in this paper could be used as a measure by which to evaluate them relative to each other or relative to a slandard. Given the capability to generate different alternative schedules, the ability to evaluate schedules then implies the ability to optimize schedules with respect to communications performance. However, it 
would seem preferable to incorporate into the scheduler itself the abllity to generate schedules directly reflecting the constraint of potential interference intervals (step (8) in the proposed procedure).

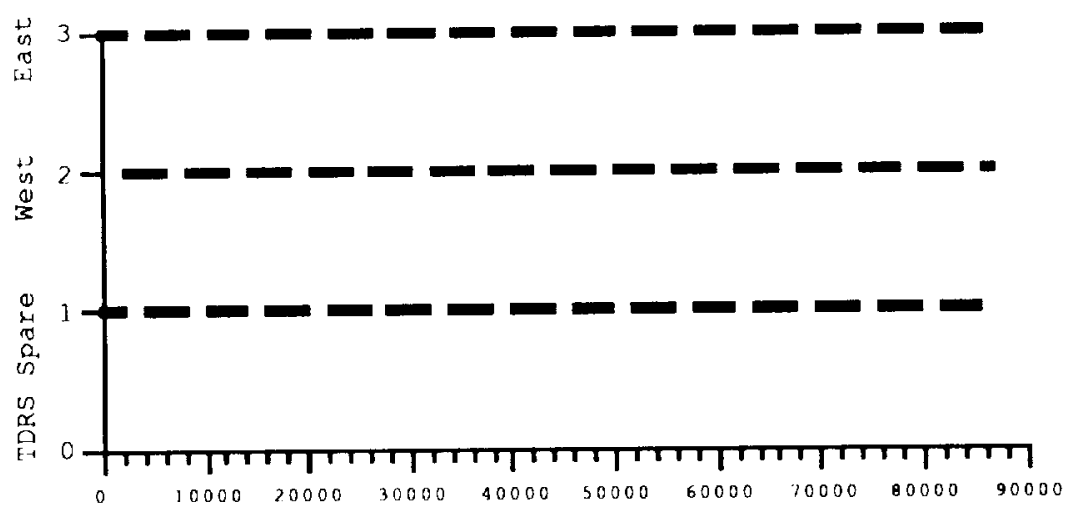

(a)

Ground Flapsed Time off set (Seconds)

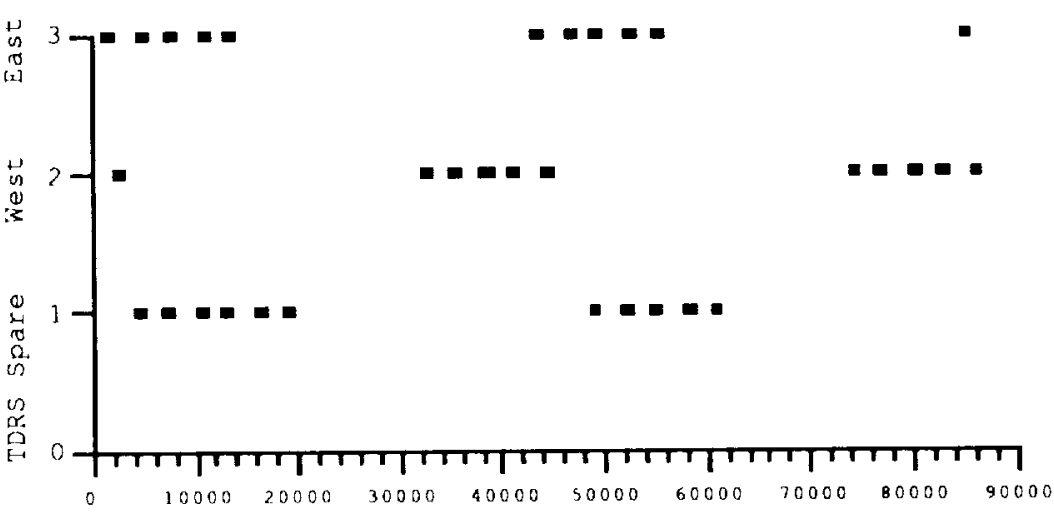

(b)

Ground Flapsed Time offset (Seconds)

Figure 6. Potential Interference Intervals at (1) TDRS Spare, (2) TDRS West, and (3) TDRS East when the desired user is SSO COLUMBIA and the interferer is Space Station. A period of twenty-four hours is represented. Each user spacecraft orbit is approximately 90 minutes in duration. (a) The users have identical orbits, so that the separation angle is always zero degrees during TDRS view periods. Thus, potential interference occupies $100 \%$ of in-view time. (b) The users have identical orbits except for a 20 degree difference in their mean anomalies. In each orbit there are two times when they are separated by less than the required separation angle: once just after appearing above the horizon as seen by the TDRS, and once just before disappearing below the horizon.

\section{Summary}

The key proposition of this paper is that an interference mitigation scheduling system (1.e., a system capable of producing schedules that are free of unacceptable interference and that minimize unnecessary restrictions on network and user resources) must reflect consideration of communications performance. The concept of using BER degradation as a function of $\mathrm{S} / \mathrm{I}$. as presented above, is a sufficient basis for an interference mitigation scheduling system.

In general, scheduling may involve any number of different user spacecraft. The scope of the approach presented in this paper is limited to the case of single interferers. The case of multiple interferers is left for future work. 
This paper presents a model of communications performance affected by the presence of mutual interference. The model formulates communications performance in terms of S/I. which is considered as a function of the interferer's angle off boresight. Required separation angles for interference mitigation can be calculated based on this functional ralationship. and these angles then can be used to determine potential interference intervals (intervals during which mutual interference could occur).

Potential interference intervals are proposed for use as a constraint by an interference mitigation scheduler. Used as a constraint, a potential interference interval disallows simultaneous communications by both of the links associated with the interval. By guaranteeing acceptable BER degradation for all desired user/interferer link combinations, except during the potentlal interference intervals associated with those link combinations. the proposed procedure guarantees schedules to be free of unacceptable mutual interference. Potential interference intervals also can be useful as the basis for evaluating and optimizing (with respect to communications performance) the user schedules produced by any scheduling system.

The method presented in this paper offers a feasible, general approach to mutual interference mitigation as a means for generating schedules free of unacceptable interference.

\section{Future Work}

A scheduling system Incorporating the approach described in this paper is being developed in the CLASS environment for use in mission planning and in communications performance optimization of user schedules. The effect of multiple interferers is to be considered in a later effort.

\section{Acknowledgments}

The authors would like to thank CLASS Project Manager Robert Godfrey of NASA/GSFC for originating the concept of an interference mitigation scheduling system for CLASS. We would also like to thank Nancy Smith Ron Vento of NASA/GSFC; Farzad Ghazvintan and Masoud Toufanian of LinCom Corporation and David Wampler of Stanford Telecommunications for valuable technical support: and our supervisor, Frank Stocklin, for his encouragement of our efforts on this project.

\section{References}

Bhargava, Vijay K., Haccoun, D., Matyas, R., \& Nuspl, P. P. (1981). Digital Communications by Satellite. New York, NY: John Wiley \& Sons.

NASA Goddard Space Flight Center (forthcoming). Space Network Support Capability Study. STDN No. 114, Revision 4. Greenbell, Maryland.

NASA Goddard Space Flight Center (1988, September). TDRS User Guide, STDN No. 101.2, Revision 6. Greenbelt, Maryland.

NASA Goddard Space Flight Center (1989. January 30). Memo, Subject: STS Link Calculations. To Distribution. From GSFC Code 531.1. Greenbelt, Maryland.

NASA Goddard Space Flight Center (1989. September). Communication Link Analysis and Simulation System. CLASS No. 101. Greenbelt, Maryland.

NASA Goddard Space Flight Center (1989, October 27). Radio Frequency Interface Control Document Between the Space Station Project and the Tracking and Data Relay Satellite System (Preliminary Baseline). Greenbelt, Maryland.

NASA Goddard Space Flight Center (1990. February). Radio Frequency Interface Control Document Between the EOS Platform and the Tracking and Data Relay Satellte System (Preliminary Baseline). Greenbelt, Maryland. 
Computer Sclences Corporation (1990, February). Network Control Center User Planning System System Requirements Document. NASA Goddard Space Flight Center, Greenbelt, Maryland.

Weltı, George, Christopher, P., \& Griffin, M. (1990, 9 March). ATDRSS Self-interference Assessment. Technical Report TR90007. Stanford Telecommunications, Inc., Reston, VA.

NASA Goddard Space Flight Center (1990, April/May). Mission Requirements and Data Systems Support Forecast. STDN No. 803. Greenbelt, Maryland.

\section{Appendix}

\section{Polarization Rejection}

Polarization rejection, $R$, of the interfering signal at the oppositely polarized TDRS SA antenna is a function of the TDRS SA antenna axial ratio $r_{w}$ (not in $\mathrm{dB}$ ) and the interferer antenna axial ratio $r_{a}$ (not in $\left.\mathrm{dB}\right)$ :

$$
\begin{aligned}
& R=10 \log \left[\frac{1+\rho_{w}^{2} \rho_{a}^{2}+2 \rho_{w} \rho_{a} \cos (2 \theta)}{\left(1+\rho_{w}^{2}\right)\left(1+\rho_{a}^{2}\right)}\right] d B \\
& \rho_{w}=\frac{r_{w}+1}{r_{w}-1}
\end{aligned}
$$

where

$$
\rho_{a}=\frac{r_{a}+1}{r_{a}-1}
$$

and where $\theta$ is the angular orientation of the electric field vector. The axial ratio is negative for right circular polarization (RCP) and posilive for left circular polarization (LCP) .

In the present application. $\theta$ is assumed to be zero degrees in keeping with the assumption of the maximum effect of the interferer.

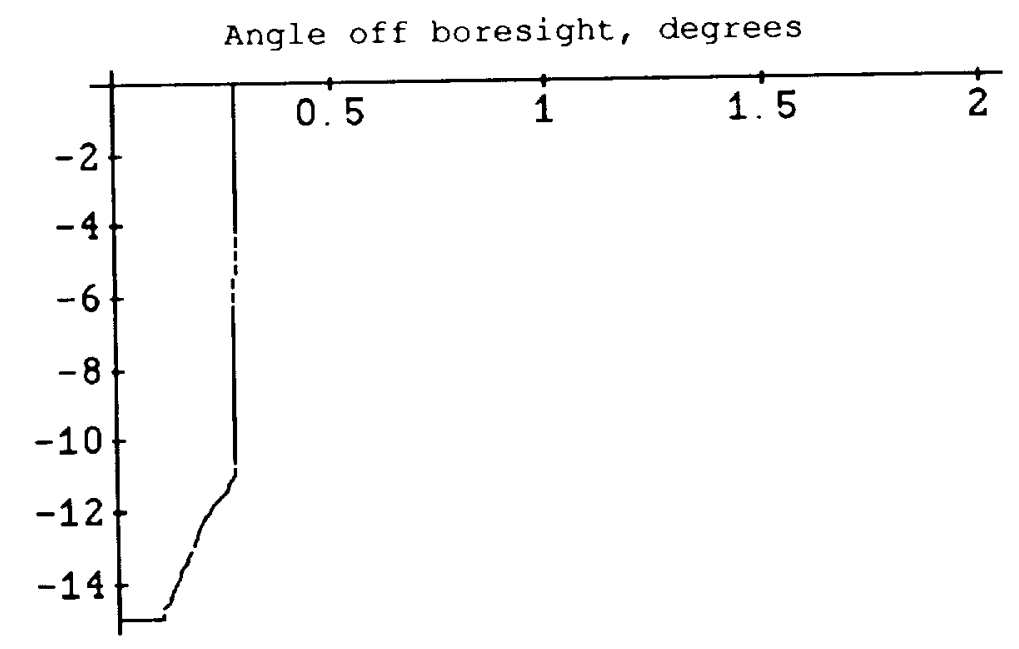

Figure A. Polarization rejection modeled as a function of angle off boresight at the TDRS SA antenna when the transmitting antenna is that of the Space Station Manned Base. 
Figure A shows the polarization rejection as a function of angle off boresight in the case of the TDRS SA antenna as the recelving antenna and the Space Station Manned Base high gain antenna as the transmitting antenna. The boresight axlal ratios are $1.0 \mathrm{~dB}$ and $2.1 \mathrm{~dB}$. respectively. Axlal ratio off boresight for the receiving antenna is modeled as described in the following section. Note that beyond the first null, since the axial ratlo is undefined, the polarization rejection is also undefined.

\section{Antenna Axial Ratio Model}

The axial ratio of the TDRS SA antenna is modeled as a function of angle off boresight. Let $a$ denote the angle off boresight at which the gain is $3 \mathrm{~dB}$ down from the boresight gain. and let $b$ denote the angle off boresight at the first null in the gain pattern. Beyond the first null, the axial ratio is undefined. The axial ratio in $\mathrm{dB}$ is then modeled as a broken straight line function of angle $\alpha$ off boresight:

$$
\begin{gathered}
r(\alpha)= \begin{cases}1 d B, & \text { if } 0 \leq \alpha \leq a \\
\left(\begin{array}{ll}
\frac{2}{b-a} \alpha+\frac{b-3 a}{b-a}
\end{array}\right) d B, & \text { if } a \leq \alpha \leq b \\
u n d e f i n e d, & \text { if } \alpha>b\end{cases} \\
\text { Note that } r(\alpha)= \begin{cases}20 \log r_{w} & \text { for the transmitting antenna } \\
20 \log r_{a} & \text { for the receiving antenna }\end{cases}
\end{gathered}
$$

This model is tllustrated in Figure B using values for $a$ and $b$ of 0.1 degrees and 0.274 degrees, respectively.

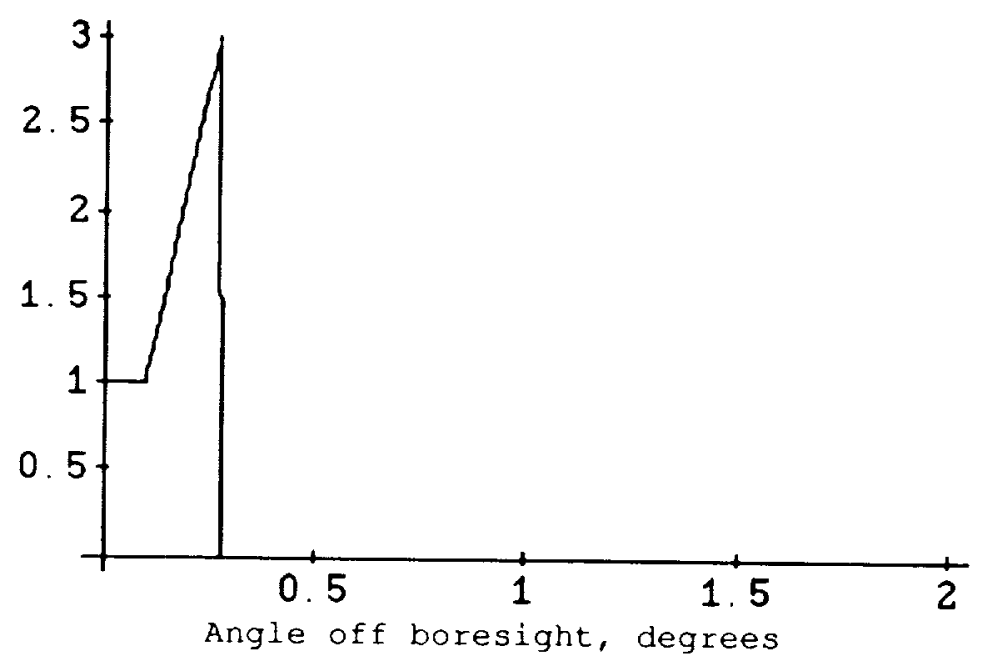

Figure B. Axial ratio modeled as a function of angle of boresight for the TDRS SA antenna. 